\title{
Emerging mosquito-borne bunyaviruses in Canada
}

\author{
Drebot $\mathrm{MA}^{1^{*}}$ \\ ${ }^{1}$ National Microbiology Laboratory, Public Health Agency of Canada, Winnipeg, MB \\ *Correspondence: mike.drebot@phac-aspc.gc.ca
}

\begin{abstract}
California serogroup and Cache Valley viruses are arboviruses (tick- and mosquito-borne pathogens) belonging to the genus Orthobunyavirus (Family Bunyaviridae). Although the majority of exposures to these viruses result in asymptomatic or mild infections, both California serogroup and Cache Valley viruses can cause febrile and neurological diseases similar in nature to those associated with infections by West Nile virus. California serogroup and Cache Valley viruses are widely distributed across North America and circulate in a number of vertebrate hosts and mosquito vectors, including several species of Aedes and other non-Culex mosquitoes. The Jamestown Canyon and snowshoe hare viruses are the most common kind of California serogroup viruses found in Canada and have been identified throughout the country. These potential pathogens may be contributing to a higher burden of illness than previously recognized and should be considered as part of the differential diagnosis for febrile and neuroinvasive disease during the mosquito season. Diagnosis can be made by requesting a diagnostic panel at the Viral Zoonoses program at the National Microbiology Laboratory. To decrease the risk of infection, education about these viruses and the importance of personal preventive measures is warranted.
\end{abstract}

\section{Introduction}

The Bunyaviridae family of RNA viruses is a very large, diverse and globally-distributed group of viruses that infect plants, vertebrates and invertebrates (1). Many medically-important bunyaviruses are vector-borne viruses that can infect rodents or arthropods. For example, hantaviruses such as Sin Nombre virus are transmitted by deer mice and cause hantavirus pulmonary syndrome $(2,3)$. Arboviruses are bunyaviruses which infect and are transmitted by ticks and mosquitoes. Mosquito-borne bunyaviruses belong to the Orthobunyavirus genus. There are approximately 170 viruses in this genus which includes 48 species and 19 serogroups. Within two of these serogroups there are four emerging viruses that are becoming increasingly recognized as important human and veterinary pathogens (1). (Text table)

\section{Four emerging arboviruses in North America in the Orthobunyavirus genus of the Bunyaviridae family ${ }^{1}$}

California serogroup: There are 17 viruses in this serogroup including:
California encephalitis virus
Inkoo virus
Jamestown Canyon virus
La Crosse virus
Snowshoe hare virus
Tahyna virus

Bunyamwera serogroup: There are 23 viruses in this serogroup including:

\section{- $\quad$ Cache Valley virus}

${ }^{1}$ The four emerging viruses are identified in bold

The California serogroup viruses circulate widely throughout the world. They include the Inkoo virus in Europe; the Tahyna viruses in Europe, Asia and Africa; and the La Crosse, snowshoe hare and Jamestown Canyon viruses in North America $(4,5)$. Several California serogroup viruses are associated with mild flu-like diseases and severe 
central nervous system infections $(4,6,7,8)$. The snowshoe hare virus has been implicated in neurological cases mainly involving children $(4,8,9)$. Another widespread California serogroup virus is the Jamestown Canyon virus which has been recently been identified as an emerging cause of febrile and neuroinvasive disease in Canada and the United States $(6,7,8,10,11,12)$. The La Crosse virus is closely related to the snowshoe hare virus and is the primary cause of viral encephalitis in children in the United States (on average 80 to 100 cases per year) and the second leading cause of arbovirus-associated neuroinvasive disease in North America $(6,13)$. No La Crosse virus associated clinical cases have been reported yet in Canada presumably due to the limited occurrence of its vectors (e.g., Aedes triseriatis and Aedes albopictus); however, future climatic changes could influence the northern expansion of these mosquito species (14).

A fourth emerging mosquito-borne orthobunyavirus is the Cache Valley virus (Bunyamwera serogroup) which circulates throughout North and South America $(15,16)$. It has been primarily associated with disease in livestock, especially sheep (15). However, patients with undiagnosed illness in western Canada have been found to harbour Cache Valley virus-specific antibody (17, Drebot unpublished findings) and several cases of neuroinvasive disease in humans caused by Cache Valley virus have been documented in the United States $(18,19)$.

There are no specific treatments or currently-available vaccines for the California serogroup and Cache Valley virus infections. Treatment for these viral infections typically includes supportive care and management of complications, such as relieving increased intracranial pressure.

The health impact of these bunyaviruses may be significantly greater than previously thought. From 1989 to 2005 , no cases of California serogroup virus infections were documented in Canada due to the discontinuation of diagnostic procedures for identifying these pathogens. As part of an enhanced approach to further develop serological assays for West Nile virus and other mosquito-borne arboviruses, new testing methodologies have been implemented and added to the existing diagnostic panels at the Viral Zoonoses program at the National Microbiology Laboratory in Winnipeg Manitoba $(11,12,20)$.

There is now sufficient evidence to indicate that when patients present with febrile and neurological disease and a history of exposure to mosquitoes, both West Nile virus and mosquito-borne bunyaviruses should be considered. In this article the ecology, epidemiology, clinical aspects, diagnostics and some recent laboratory-based surveillance data of California serogroup virus and Cache Valley virus will be discussed.

\section{California serogroup viruses}

The La Crosse, snowshoe hare and Jamestown Canyon viruses are the main emerging and neglected California serogroup viruses in North America $(4,6,7,9,11,13)$. Humans may acquire infection through mosquito bites resulting in asymptomatic to mild febrile illness (with fever, chills, abdominal pain, cough, headache and photophobia) and acute central nervous system infection (meningitis and/or encephalitis). Although most patients with California serogroup viral encephalitis recover fully, some long-standing neurologic sequelae have been reported, in particular for the La Crosse virus (13).

In Canada, California serogroup virus activity has been demonstrated in all provinces and territories $(8,9,11,12)$. From 1978 to 1989, 23 cases of symptomatic infection were diagnosed in Canada, the majority of which were snowshoe hare viruses (18 cases) with three cases of Jamestown Canyon virus and two California serogroup viruses of unknown identity (8, Artsob and Drebot, unpublished findings).

The risk for California serogroup virus exposure extends from May to October, as the predominant vectors carrying the snowshoe hare and Jamestown Canyon viruses are (unlike West Nile virus) non-Culex mosquitoes such as Aedes, Culiseta and Anopheles species $(4,7,8)$. As well, the amplifying hosts / reservoirs for these viruses are either small mammals such as squirrels, chipmunks, hares and various rodents (snowshoe hare viruses), or larger animals such as deer and elk (Jamestown Canyon viruses) (Figure 1). Livestock such as horses, cattle and sheep also exhibit significant levels of seroprevalence, however, they probably do not contribute significantly to the enzootic transmission cycle of these viruses due to low viremia (21, Drebot, unpublished findings). Transovarial transmission is the most likely overwintering mechanism which involves 
infected mosquitoes transmitting virus to their offspring in the egg where the snowshoe hare and Jamestown Canyon viruses overwinter.

Figure 1: California serogroup (Jamestown Canyon, snowshoe hare viruses) and Cache Valley virus transmission cycles

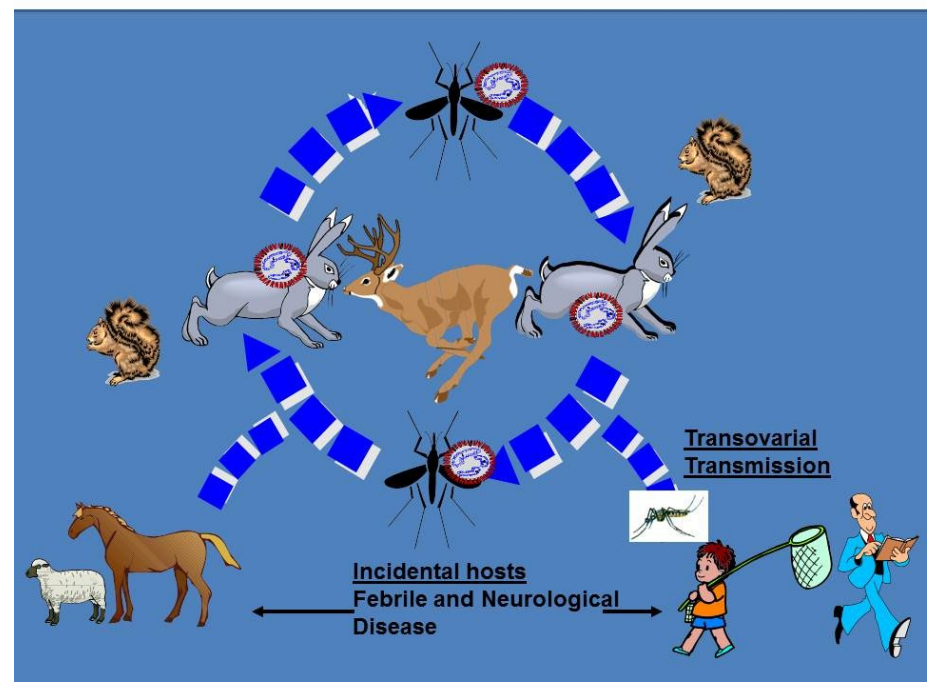

The presence of both vectors and reservoirs throughout woodlands and parks in both rural and urban areas increases the possibility of significant levels of virus circulation not only in southern parts of Canada but also in northern locations such as the Yukon, North West Territories and Alaska. As a result, there is risk for human and animal exposures during the entire mosquito season and over a wide geographic area.

\section{Snowshoe hare virus}

The snowshoe hare virus circulates widely across Canada and the United States in enzootic cycles involving nonCulex mosquitoes and mammals such as hares and squirrels (8). It was first isolated in 1958 from the serum of a snowshoe hare (Lepus americanus) in Montana. Human disease caused by this virus was initially documented in Canada in 1978 when three encephalitis infections were diagnosed in Québec (three boys aged 7, 6 and 10 years old with symptoms of fever, nausea, vomiting, headache, confusion and agitation) and one case of meningitis identified in Ontario (30 year old male) (8). Most of the snowshoe hare virus cases were associated with neuroinvasive diseases such as encephalitis and meningitis and predominately involved children, a similar epidemiology that is observed for the closely related La Crosse virus which is found in the eastern and Midwestern United States (13).

\section{Jamestown Canyon virus}

The Jamestown Canyon virus was initially isolated in the United States in 1961 from a pool of Culiseta inornata mosquitoes collected in Jamestown Canyon near Boulder, Colorado (4). Jamestown Canyon virus infections may cause a similar range of diseases as observed for the snowshoe hare virus including both febrile and acute central nervous system infection $(4,7)$. Respiratory system involvement has been observed in a number of patients. In contrast to the snowshoe hare virus, most Jamestown Canyon virus-infected individuals with severe symptomatic disease are adults and their primary reservoirs appear to be deer and related ruminants (7).

The Jamestown Canyon virus was thought to be primarily concentrated in eastern North America, but recent reports have suggested that the geographic distribution of human Jamestown Canyon virus infection is wider than previously recognized (7). Based on serosurveys in Canada and the United States it is estimated that approximately $25 \%$ of the population may have antibodies to the Jamestown Canyon virus but the actual seroprevalance rates may range from $1 \%$ to $40 \%$ or greater for both the Jamestown Canyon and snowshoe hare viruses depending upon the region $(22,23,24$, Drebot unpublished data). The estimated ratio of asymptomatic to 
symptomatic infections is believed to be in the range of 100:1 to 1500:1 based on studies involving the related La Crosse virus (25). Many human infections of the Jamestown Canyon virus may go undetected because of its nonspecific clinical presentation and limited availability of sensitive tests for the agent.

\section{Clinical symptoms}

The most frequent symptoms include headache, fever, dizziness and vomiting, while photophobia, respiratory distress and rash are also observed $(25,26)$. When the central nervous system is affected, clinical syndromes ranging from febrile headache, muscle weakness to aseptic meningitis to encephalitis may occur and these are usually indistinguishable from similar syndromes caused by other viruses. California serogroup viral meningitis is characterized by fever, headache, stiff neck and pleocytosis and infections involving children may result in seizures. Snowshoe hare / Jamestown Canyon virus associated encephalitis is characterized by fever, headache and altered mental status ranging from confusion to coma with or without additional signs of brain dysfunction (7, $8,26,27)$. Severe California serogroup infections result in a variety of sequelae such as behaviour changes, learning disabilities and cognitive deficits. $(25,26)$

\section{Laboratory diagnostics}

California serogroup virus serological procedures such as the IgM enzyme-linked immunosorbent assay (ELISA) and plaque reduction neutralization test (PRNT) are the primary testing methodologies used to diagnose California serogroup infections $(7,11,12,13)$. Acute and convalescent sera from suspected cases are recommended for determining the diagnostic rise or decrease in California serogroup virus specific antibody titres and documenting seroconversions. For neurological disease cases, samples of cerebrospinal fluid should be included for detection of acute IgM antibody or viral genomic sequences (by the polymerase chain reaction) which would also constitute confirmatory laboratory evidence of an infection associated with accompanying clinical characteristics $(11,12,26)$. However, it should be noted that it is quite rare to detect the snowshoe hare or Jamestown Canyon viruses in both brain biopsy tissue and the cerebrospinal fluid either by polymerase chain reaction or isolation (26).

As observed for West Nile virus infections, there is evidence that IgM may persist for several months or even years in sera from patients exposed to California serogroup viruses (27). As a result, lingering IgM may confound the diagnostics used in identifying current cases of California serogroup illness when positive serology is documented using only acute samples of sera.

Case definitions for California serogroup viruses (Snowshoe hare and Jamestown Canyon viruses)

A "confirmed" case is based on any of the following laboratory criteria:

- Fourfold or greater change in virus-specific antibody titre

- $\quad$ Presence of either virus-specific IgM or neutralization antibodies in cerebrospinal fluid

- Detection of virus-specific RNA in cerebrospinal fluidor isolation of virus by cell culture (rarely observed)

A "probable" case includes:

- An individual with a clinically compatible illness (and symptoms observed during the mosquito season) and detectable snowshoe hare / Jamestown Canyon virus IgM antibody and virus specific neutralization antibodies in the acute serum sample

\section{Laboratory-based surveillance}

The National Microbiology Laboratory developed serological platforms to test for California serogroup virus infections using IgM ELISAs and PRNTs in 2005 following a period when there were no diagnostic procedures for identifying these pathogens $(9,11)$. By incorporating these assays for testing suspect cases of non-West Nile virus mosquito-borne agents, the first California serogroup infection in over 15 years was identified involving a pediatric snowshoe hare virus case in Nova Scotia in 2006 (9).

Since 2006, over 200 "probable" and "confirmed" cases of snowshoe hare and Jamestown Canyon virus infections have been documented including confirmed cases of neurological disease in various provinces across Canada (11, 12; Drebot, unpublished findings). Detailed clinical and diagnostic workups and case identification involved one patient in British Columbia, three patients in Alberta, one patient in Manitoba, one patient in Québec, 
one patient in New Brunswick and one patient in Nova Scotia (10, Drebot, unpublished findings). The majority of probable and confirmed cases have been associated with the Jamestown Canyon virus $(70 \%)$ which is in contrast to what was previously observed in the 70 and 80 's when most cases of California serogroup virus infection in Canada associated with the snowshoe hare virus (8). It is unclear whether this is due to a change in virus circulation or abundance or is due to improved sensitivity and specificity of serological diagnostics. Grimstead et al have indicated that previously-used $\mathrm{HI}$ assays may not have been sensitive enough to detect Jamestown Canyon virus exposures as compared to currently employed serological methods such as IgM ELISAs (24). The preponderance of the Jamestown Canyon virus in recent serosurveys is also consistent with this virus being the California serogroup agent the majority of individuals are exposed to in Canada $(11,12,22,23$, Drebot, unpublished findings).

\section{Cache Valley virus}

The Cache Valley virus is another mosquito-borne orthobunyavirus that is also primarily transmitted by non-Culex mosquitoes. Similar to the Jamestown Canyon virus, its main animal reservoir / amplifying host is believed to be deer $(15,16)$. The Cache Valley virus was first isolated in 1956 in Cache Valley, Utah, USA but is endemic throughout Canada, the United States, the Caribbean, Mexico and Argentina $(15,28,29)$. While the Cache Valley virus can infect humans as well as a wide variety of livestock, clinical disease has been primarily documented in sheep (15). Most natural infections in non-pregnant sheep are subclinical; however, the virus may cross the placenta in pregnant ewes and infect the fetus resulting in the birth of diseased lambs with malformations observed in the musculoskeletal and central nervous systems. The Cache Valley virus has been suspected in past sheep outbreaks in Canada based on positive serology among ewes in farm flocks but in 2012 and 2013, Cache Valley virus infections in livestock were verified by viral isolation and tissue positive PCR for the first time in Ontario and Québec $(30,31)$. Seroprevalence studies have demonstrated seropositivity rates of up to $40 \%$ among sheep and other ruminants at various sites in Saskatchewan, Ontario and Québec.

\section{Clinical cases}

Human infections with the Cache Valley virus appear to be quite common in areas where the virus is enzootic and seroprevalence rates in humans may be as high as $18 \%(28)$. Although human neuroinvasive illness has rarely been diagnosed, there have been three reports of severe Cache Valleyviral associated disease in the United States including a fatal case of encephalitis $(18,19)$. The low frequency of cases is presumably due to the fact that laboratories rarely test for the virus and cases involving febrile and neuroinvasive disease may be undiagnosed. Recent serological testing of West Nile virus suspect-cases from Manitoba and Saskatchewan identified Cache Valley virus exposures in 5 to $16 \%$ of patients screened for viral specific antibody (17). As well, the strain of Cache Valley virus isolated from a recent human case in the US was almost identical to the isolates obtained during the sheep outbreaks in Québec and Ontario indicating that currently circulating genotypes of the virus do exhibit the potential for pathogenicity in humans and other animals (30). The Cache Valley virus has also been associated with congenital defects in humans (i.e., macrocephaly in infants), but the specific role that this virus may play in inducing these abnormalities has not been determined and further verification is warranted (32).

\section{Laboratory diagnosis}

There is no commercial diagnostic kit available for identifying cases of the Cache Valley virus and antibodies to the agent will not cross react significantly in California serogroup assays. The National Microbiology Laboratory conducts neutralization tests to identify Cache Valley virus-specific antibodies in sera and has viral isolation procedures in place as well (17). IgM and IgG ELISAs are currently in development for the Cache Valley virus to facilitate case detection. Given the wide ranging nature of the potential pathogen in Canada and the increasing identification of cases among livestock in the country, the potential for neuroinvasive cases among patients during the mosquito season is low but remains possible. 


\section{Discussion}

Emerging and neglected mosquito-borne bunyaviruses such as the California serogroup and Cache Valley viruses may be contributing to a significant number of cases of undiagnosed febrile and neuroinvasive disease during the Canadian mosquito season. Recent documentation of Jamestown Canyon and snowshoe hare virus cases indicate that these viruses are contributing to significant morbidity when mosquitoes are prevalent. A recent study by Kulkarni et al (33) used spatial and temporal statistics to identify seasonal clusters of Canadian hospitalizations and suggested arboviral agents in addition to West Nile virus may be implicated as undetermined aetiologies of neurological disease. It should be noted that the seasonal and geographic risk for exposure for mosquito-borne bunyaviruses is more widespread than West Nile virus.

Significant numbers of arthropod-borne bunyavirus infections are likely being undetected due to a lack of commercially-available diagnostic assays and low-level surveillance. Currently the National Microbiology Laboratory is the only laboratory in Canada to perform California serogroup virus testing. Only one commercial serological assay for California serogroup viruses exists and it is primarily used as an immunofluorescent test for La Crosse virus antibody (20). Although serological cross reactivity between the snowshoe hare, Jamestown Canyon and La Crosse viruses may occur, recent studies have shown that La Crosse virus-specific diagnostic platforms may not always detect antibodies to other California serogroup viruses and cases may be missed (20). Further development and implementation of commercial and "in house" kits for a wider variety of orthobunyaviruses may aid in detecting additional cases associated with these viruses. Improved and timely diagnostics will aid clinicians in making patient-care and management decisions.

Disease prevention is primarily achieved through public education. Personal risk reduction measures include decreasing the risk of mosquito bites by avoiding exposure, wearing protective clothing and using insect repellent. The elimination of mosquito breeding sites to prevent arboviral infection is also recommended.

\section{Conclusion}

Febrile and neurologic illness may be caused by emerging mosquito-borne bunyaviruses in Canada. Clinicians should consider California serogroup and Cache Valley viral infections in the differential diagnoses when an arboviral infection is suspected and testing for West Nile virus is inconclusive. Enhanced surveillance and the utilization of a wider panel of diagnostic assays could lead to the further identification of neuroinvasive disease caused by these emerging viruses. In the meantime, education about these viruses and the importance of personal preventive measures to decrease the risk of infection are warranted.

\section{Acknowledgements}

The author wishes to acknowledge the assistance of Canada's Public Health Laboratories and the provincial veterinarian labs who were involved in the identification of bunyavirus cases associated with California serogroup and Cache Valley virus infection. The author also acknowledges Dr. Harvey Artsob for sharing unpublished findings involving California serogroup virus cases as well as the excellent technical expertise and diagnostic testing contributions of Kai Makowski, Kristina Dimitrova, Kimberly Holloway and Maya Andonova.

\section{Conflict of interest}

None.

\section{References}

(1) King AMQ, Lefkowitz E, Adams MJ, Carstens EB. Family bunyaviridae. In: King AMQ, Adams MJ, Carstens EB, Lefkowitz EJ, editors. Virus Taxonomy: Ninth Report of the International Committee on Taxonomy of Viruses. San Diego: Elsevier; 2012. p. 725741.

(2) Kruger DH, Figueiredo LT, Song JW, Klempa B. Hantaviruses--globally emerging pathogens. J Clin Virol. 2015 Mar; 64:128-36. 
(3) Drebot MA, Jones S, Golla A, Safronetz D, Strong JE, Kobinger G, Lindsay RL. Hantavirus pulmonary syndrome in Canada: An overview of clinical features, diagnostics, epidemiology, and prevention. Can Commun Dis Rep. 2015 June;41(6).

(4) Leduc JW. Epidemiology and ecology of the California serogroup viruses. Am J Trop Med Hyg. 1987;37(3) Suppl: 60S-68S

(5) Putkuri N, Kurkela S, Levanov L, Huhtamo E, Vaheri A, Sironen T, Vapalahti O. Isolation and characterization of a California encephalitis serogroup orthobunyavirus from Finnish mosquitoes. Infect Genet Evol. 2014;22:164-73.

(6) Lindsey NP Lehman JA, Staples JE, Fischer M. West Nile virus and other arboviral diseases - United States, 2013. MMWR. 2014;63(24):521-526.

(7) Lowell J, Higgins DP, Drebot M, Makowski K, Staples E. Human Jamestown Canyon virus infection --- Montana 2009. MMWR. 2011;60(20):652-655.

(8) Artsob H. Arbovirus activity in Canada. Arch Virol. 1990;(Suppl 1):249-58.

(9) Meier-Stephenson V, Langley JM, Drebot M, Artsob H. Encephalitis in the summer: A case of Snowshoe hare (California serogroup) virus infection in Nova Scotia. CAN COMMUN DIS REP. 2007;33(11):23-6.

(10) Drebot M.A. A laboratory-confirmed case of Jamestown Canyon virus encephalitis in a Quebec resident with travel history to Maine and New Hampshire. Am J Trop Med Hyg. 2012;87(5)(Suppl 1):280.

(11) Makowski K, Dimitrova K, Andonova M, Drebot M. An overview of California serogroup virus diagnostics and surveillance in Canada in 2008. Int J Antimicrob Agents. 2009;34:S19.

(12) Makowski K, Dimitrova K, Andonova M, Holloway K, Kadkhoda K, Van Caeseele P, Drebot M. The identification of probable cases of California serogroup virus infections in Manitoba in 2010. Can J Infect Dis Med Microbiol. 2011;22:15A-6A.

(13) Gaensbauer JT, Lindsey NP, Messacar K, Staples JE, Fischer M. Neuroinvasive arboviral disease in the United States: $2003-2012$. Pediatrics. 2014;134(3);e642-650.

(14) Mechai S, Margos G, Feil EJ, Lindsay LR, Ogden NH. Recent and projected future climatic suitability of North America for the Asian tiger mosquito Aedes albopictus. Appl Environ Microbiol. 2014 Dec 12. pii: AEM.03730-14. [Epub ahead of print].

(15) de la Concha-Bermejillo, A. Cache Valley virus is a cause of fetal malformation and pregnancy loss in sheep. Small Ruminant Res. 2003;49:1-9.

(16) Andreadis TG Armstrong PM, Anderson JF, Main AJ. 2014 Spatial-temporal analysis of Cache Valley virus (Bunyaviridae: Orthobunyavirus) infection in Anopheline and Culicine mosquitoes (Diptera: Culicidae) in the Northeastern United States, $1997-$ 2012. Vector Borne Zoonotic Dis. 2014;14(10):763-773.

(17) Dimitrova K, Andonova M, Makowski K, Holloway K, Levett PN, Kadkhoda K, Drebot M. Preliminary evidence of Cache Valley virus infections and associated human illness in western Canada in 2009. Can J Infect Dis Med Microbiol. 2011;22:15A

(18) Sexton DJ, Rollin PE, Breitschwerdt EB, Corey GR, Myers SA, Dumais MR.et al. Life-threatening Cache Valley virus infection. N Engl J Med. 1997;336:547-9.

(19) Nguyen NL, Zhao G, Hull R, Shelly MA, Wong SJ, Wu G et al. Cache Valley virus in a patient diagnosed with aseptic meningitis. J Clin Microbiol. 2013;51:1966-9.

(20) Makowski K, Dimitrova K, Andonova M, Drebot M. Assessing serological cross-reactivity among California serogroup viruses using an IgM ELISA platform. Can J Infect Dis Med Microbiol. 2010;21:26A.

(21) Goff G, Whitney H, Drebot MA. Roles of host species, geographic separation and isolation in the seroprevalence of Jamestown Canyon and Snowshoe hare viruses in Newfoundland. Appl Environ Microbiol. 2012 Sep;78(18):6734-40.

(22) Adjemian J, Weber IB, Quiston J, et al. Zoonotic infections among employees from Great Smoky Mountains and Rocky Mountain National Parks, 2008-2009. Vector Borne and Zoonotic Dis. 2012;12(11):922-931.

(23) Sampasa-Kanyinga H, Levesque B, Anassour-Laouan-Sidi E, et al. Zoonotic infections in communities of the James Bay Cree territory: An overview of seroprevalence. Can J Infect Dis Med Microbiol. 2013;24(2):79-84.

(24) Grimstad PR, Calisher CH, Harroff RN, Wentworth BB. Jamestown Canyon virus (California serogroup) is the etiological agent of widespread infection in Michigan humans. Am J Trop Med Hyg. 1986;35(2):376-386.

(25) Haddow AD, Odol A. The incidence risk, clustering and clinical presentation of La Crosse virus infections in eastern United States, 2003 - 2007. PLOS One. 2009;4(7):e6145.

(26) Huang C Campbell W, Grady L, Kirouac I, LaForce FM. Diagnosis of Jamestown Canyon encephalitis by polymerase chain reaction Clin Infect Dis. 1999;28:1294-7.

(27) Makowski K, Dimitrova K, Andonova M, Vancaeseele P, Dawood M, Drebot M. IgM persistence: A diagnostic concern for identifying cases of California serogroup virus infection. Can J Infect Dis Med Microbiol. 2010;21:26A-7A.

(28) Blitvich BJ, Saiyasombat R, Talavera-Aguilar LG, Garcia-Rejon JE, Farfan-Ale JA, Machain-Williams C, Loroño-Pino MA. Orthobunyavirus antibodies in humans, Yucatan Peninsula, Mexico. Emerg Infect Dis. 2012;18(10):1629-1632.

(29) Blitvich BJ, Saiyasombat R, Travassos da Rosa A, Tesh RB, Calisher CH, Garcia-Rejon JE, Farfán-Ale JA, Loroño RE, Bates A, Loroño-Pino MA. Orthobunyaviruses: A common cause of infection of livestock in the Yucatan peninsula of Mexico. Am J Trop Med Hyg. 2012 Dec;87(6):1132-9.

(30) Shapiro J, Brooks A, Menzies P, Rau J, Drebot M, Andonova M et al. Cache Valley virus identified as a cause of malformed lambs in Ontario. AHL News. 2012;16:15.

(31) MAPAQ. Cas de malformations congenitales chez des ovins causees par le virus de la Vallee Cache. Info-RAIZO. 2013;1.

(32) Calisher $\mathrm{CH}$, Sever JL. Are North American bunyamwera serogroup viruses etiologic agents of human congenital defects of the central nervous system? Emerg Infect Dis. 1995 Oct-Dec;1(4):147-51.

(33) Kulkarni MA, Lecocq AC, Artsob H, Drebot MA, Ogden NH. Epidemiology and aetiology of encephalitis in Canada, 1994-2008: A case for undiagnosed arboviral agents? Epidemiol Infect. 2013;141:2243-2255. 